\section{Supporting FAIR data discoverability in clinical research: Building an object metadata repository (MDR) of clinical study objects}

\author{
C. Ohmann ${ }^{1}$, S. Goryanin ${ }^{1}$, S. Canham¹, J. Kudzia², S. Nicotri³ ${ }^{3}$ A. Italiano³, G. Donvito ${ }^{3}$, L. Dutka², \\ S. Battaglia ${ }^{1}$, M. Orzechowski², M. Borzecki ${ }^{2}$ \\ ${ }^{1}$ ECRIN, ${ }^{2}$ ACK Cyfronet AGH, ${ }^{3}$ Istituto Nazionale di Fisica Nucleare (INFN)
}

\section{Background:}

Data and documents generated in a clinical study (study protocol, statistical analysis plan, case report form, analysis data set, publication, etc.) is collected and stored in a wide variety of different locations (e.g. data repositories, institutional repositories, trial registries, publications, web pages, etc.). To maximize the discoverability of all these data objects, it is necessary to collect the metadata about them, including object provenance, location and access details, into a single system (Figure 1).

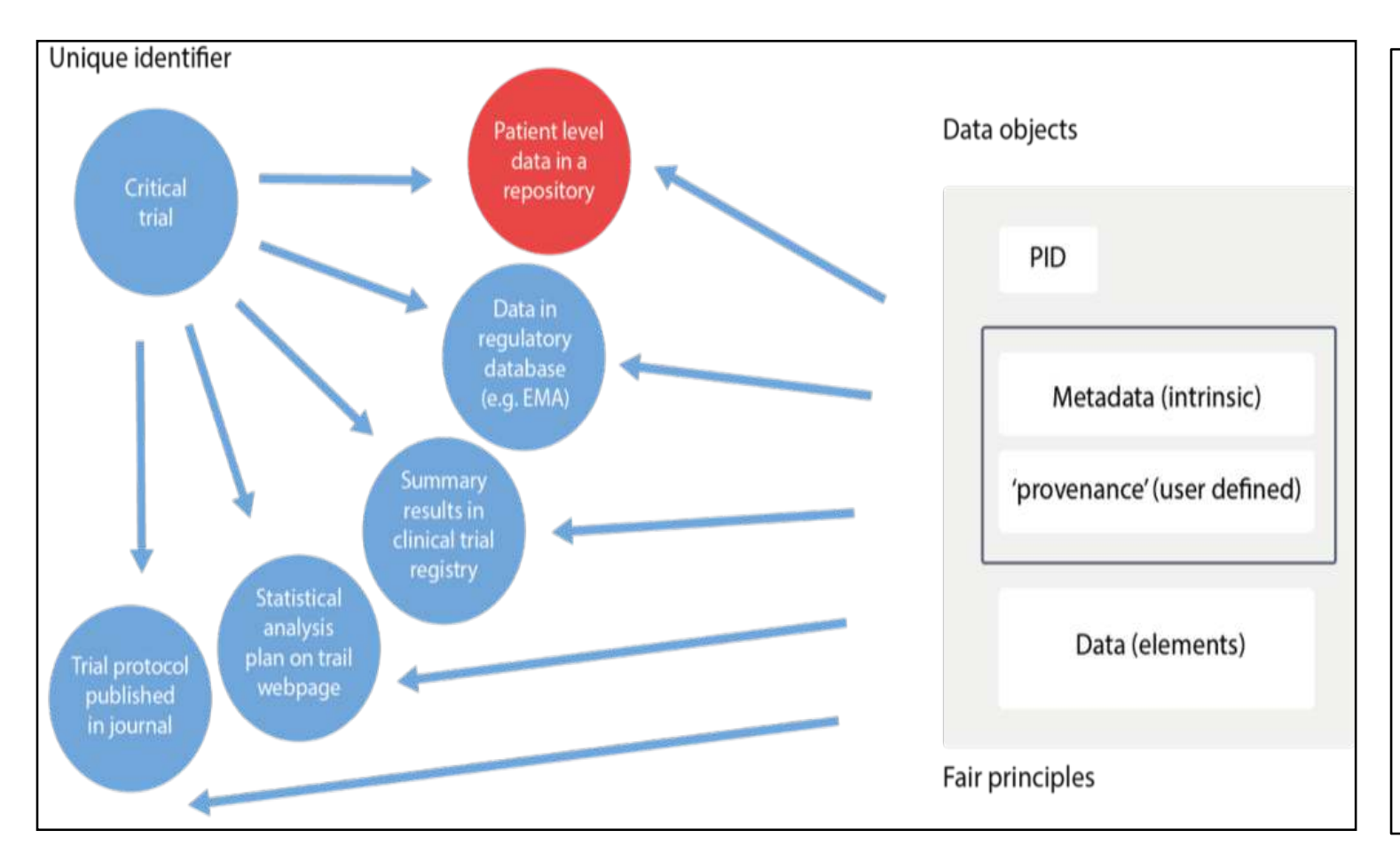

\section{Target:}

In the EU H2O2O funded XDC project (http://www.extremedatacloud.eu/) we developed an MDR (MetaData Repository) to standardise, assemble and display the metadata about clinical studies and related data objects and provide access to them through a web portal.

Fig. 1 - MDR schema: Collection, storage and linkage of clinical trial studies and related data objects from different locations

\title{
Conclusion:
}

A demonstrator MDR has been successfully developed and tested. Next steps will be to integrate further data sources and to increase functionality. It is planned to provide the MDR as open service in the European Open Science Cloud (EOSC).

\section{References:}

1. Canham, Steve. (2019). ECRIN Clinical Research Metadata Schema Version 3.0 (November 2019) (Version 3.0). Zenodo.

2. Goryanin et al.: Supporting FAIR data discoverability with a metadata repository for clinical study objects EOSC-hub Week 2019

\section{Implementation:}

The implementation process is presented in Figure 2. Metadata of clinical studies and related data objects were collected from 4 sources, processed, stored and mapped to a common metadata schema by ECRIN (Table 1). The metadata were converted into JSON format and transferred and injected into the Onedata environment. A harvester built upon Elastic search was developed by INFN and implemented as plugin into OneData. The web portal for searches of studies and metadata of associated data objects was provided by Onedata (Figure 2,3).

\begin{tabular}{|c|c|c|c|c|}
\hline Source & Extraction Method & $\begin{array}{l}\text { Study } \\
\text { Records }\end{array}$ & $\begin{array}{l}\text { Data } \\
\text { Object } \\
\text { Records }\end{array}$ & $\begin{array}{l}\text { Study- } \\
\text { object } \\
\text { links }\end{array}$ \\
\hline $\begin{array}{l}\text { Clinical } \\
\text { Trials.gov }\end{array}$ & $\begin{array}{l}\text { Bulk download of } x \mathrm{l} l \\
\text { files, extraction from } \\
\text { the } x m l\end{array}$ & 325,352 & 389,665 & 389,665 \\
\hline PubMed & $\begin{array}{l}\text { Filtered download of } \\
\text { xml files via API, } \\
\text { extraction from the xml }\end{array}$ & 0 & 79,392 & 85,855 \\
\hline BioLINCC & $\begin{array}{l}\text { Web scraping from the } \\
\text { BioLINCC web site }\end{array}$ & 203 & 1337 & 1337 \\
\hline Yoda & $\begin{array}{l}\text { Web scraping from the } \\
\text { Yoda web site }\end{array}$ & 376 & 2544 & 2544 \\
\hline Total & & 325,931 & 472,938 & 479,401 \\
\hline \multicolumn{5}{|c|}{ MDR Metadata Repostion for cinincal trial data objects } \\
\hline 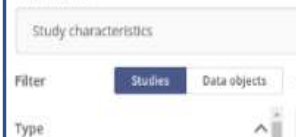 & $v$ enerer & & & $-m=0$ \\
\hline 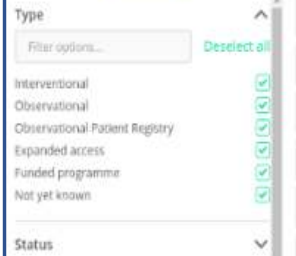 & 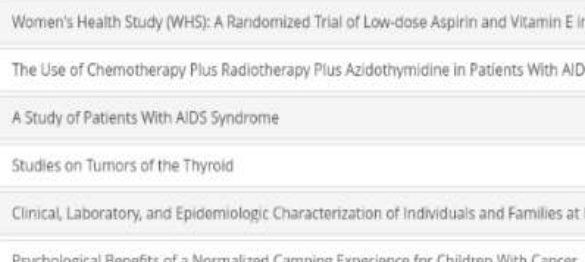 & 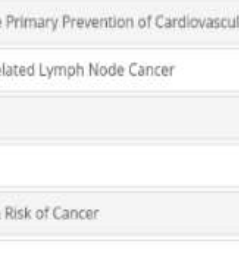 & & \\
\hline & 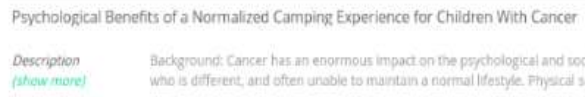 & 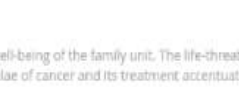 & 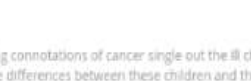 & wetentisys \\
\hline 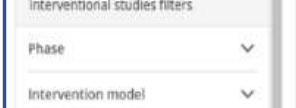 & 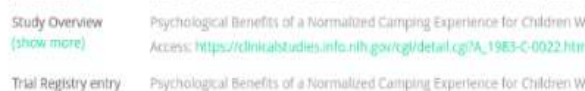 & matomenter & & $\bullet$ \\
\hline 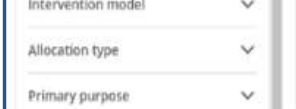 & ins & & & \\
\hline 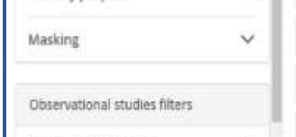 & 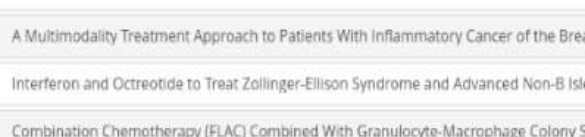 & 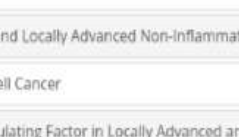 & & ${ }^{*}$ \\
\hline 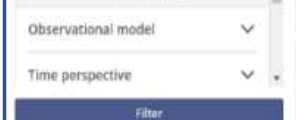 & 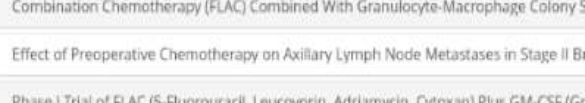 & 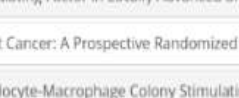 & & 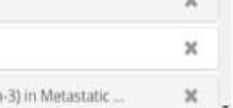 \\
\hline & 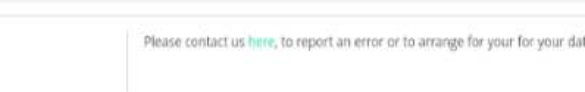 & atamontever & ONEJATA & Cxc Aosc \\
\hline
\end{tabular}

Table 1-Records downloaded and extracted

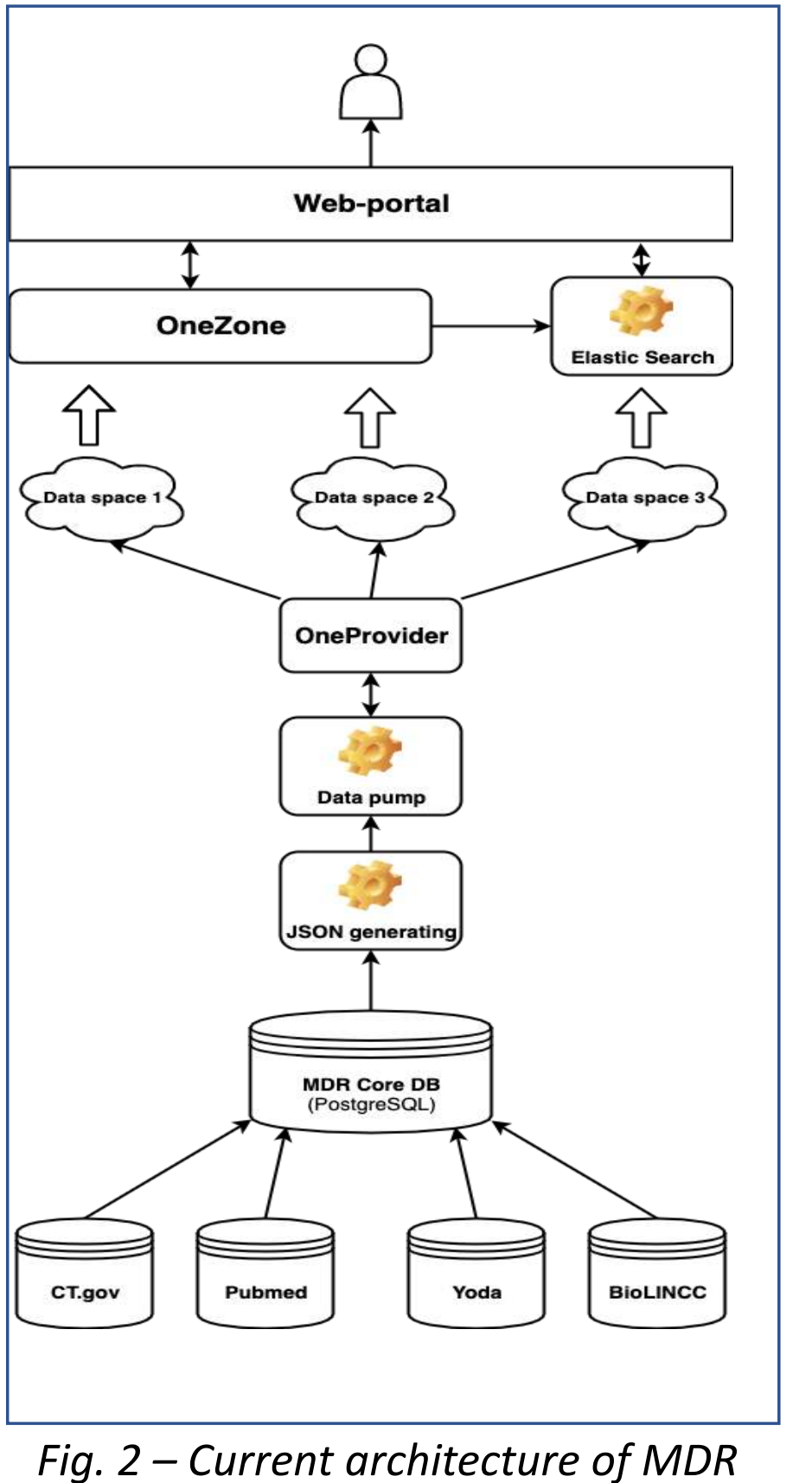

Fig. 2 - Current architecture of MDR demonstrator

Fig. 3 -Screenshot of MDR portal 